\title{
What happens when doctors are patients? Qualitative study of GPs
}

\author{
Fiona Fox, Michael Harris, Gordon Taylor, Karen Rodham, Jane Sutton, \\ Brian Robinson and Jenny Scott
}

\author{
ABSTRACT

\section{Background} \\ Current evidence about the experiences of doctors who \\ are unwell is limited to poor quality data. \\ Aim \\ To investigate GPs' experiences of significant illness, \\ and how this affects their own subsequent practice. \\ Design of study \\ Qualitative study using interpretative phenomenological \\ analysis to conduct and analyse semi-structured \\ interviews with GPs who have experienced significant \\ illness.

\section{Setting} \\ Two primary care trusts in the West of England. \\ Method \\ A total of 17 GPs were recruited to take part in semi- \\ structured interviews which were conducted and \\ analysed using interpretative phenomenological \\ analysis

\section{Results} \\ Four main categories emerged from the data. The \\ category, 'Who cares when doctors are ill?' embodies \\ the tension between perceptions of medicine as a \\ 'caring profession' and as a 'system'. 'Being a \\ doctor-patient' covers the role ambiguity experienced \\ by doctors who experience significant illness. The \\ category 'Treating doctor-patients' reveals the fragility \\ of negotiating shared medical care. 'Impact on \\ practice' highlights ways in which personal illness can \\ inform GPs' understanding of being a patient and their \\ own consultation style.

\section{Conclusion} \\ Challenging the culture of immunity to illness among \\ GPs may require interventions at both individual and \\ organisational levels. Training and development of \\ doctors should include opportunities to consider \\ personal health issues as well as how to cope with role \\ ambiguity when being a patient and when treating \\ doctor-patients. Guidelines about being and treating \\ doctor-patients need to be developed, and GPs need \\ easy access to an occupational health service.

\section{Keywords} \\ doctor-patients; general practitioners; qualitative \\ interview.
}

\section{INTRODUCTION}

High levels of work-related stress along with susceptibility to anxiety, depression, alcoholism, and substance abuse make GPs vulnerable to significant illness. ${ }^{1-3}$ Despite the quantity of expert opinion and anecdotal reports, current evidence about the experiences of doctors who are unwell is limited to poor quality data. ${ }^{4}$ Ten studies that took a qualitative approach to exploring the barriers to seeking health care have been identified. ${ }^{3,5-13}$ Many of these studies used postal questionnaires,,$^{3,7-9}$ or face-to-face questionnaires $^{12}$ to elicit attitudes and opinions of large numbers of participants. A few studies employed mixed methodology designs, including a survey and focus groups, ${ }^{10}$ and focus groups and interviews. ${ }^{6}$ Just two studies employed face-to-face interviews to explore the experiences of smaller numbers of participants. ${ }^{5,11}$ Of the qualitative studies based on UK samples, just three recruited 'family physicians', ${ }^{6,7,12}$ while others focused on medical graduates ${ }^{3}$ or doctors. ${ }^{5}$ This indicates that there is a paucity of evidence-based research which explores the subjective experiences of GPs in the UK who become ill.

F Fox, BSc(Hons), PhD, research fellow; M Harris, FRCGP, MMEd, GP principal, Hope House Surgery, Radstock. G Taylor, BSc(Hons), MSc, PhD, senior lecturer in medical statistics, Research and Development Support Unit, School for Health; K Rodham, BSc(Hons), PhD, lecturer in health psychology, Department of Psychology; J Sutton, BSc(Hons), PhD, MRPharms (IP), occupational psychologist and teaching fellow in pharmacy practice; J Scott, BSc(Hons), PhD, senior lecturer in pharmacy practice \& medicines use, University of Bath, Bath. B Robinson, PhD, FRCPsych, consultant psychiatrist, SBW Centre, Bristol General Hospital, Bristol.

Address for correspondence

Dr Michael Harris, Hope House Surgery, The Street, Radstock, BA3 3PL.

E-mail: michaelharris681@btinternet.com

Submitted: 30 March 2009; Editor's response: 15 May 2009; final acceptance: 17 September 2009.

(C)British Journal of General Practice 2009; 59: 811-818.

DOI: 10.3399/bjgp09X472872 


\section{How this fits in}

The perceived need to portray an unrealistically healthy image can prevent GPs from acknowledging illness in themselves. The professional status of doctors and the organisation of primary care reinforce a culture in which the distress of doctors who are ill is overlooked. When doctors do become patients, shared management of the illness is often problematic. Role ambiguity and anxiety are experienced by GPs both when they are seeking health care from and when providing health care to other doctors. Participants reported their perceptions that an experience of personal illness can inform or alter subsequent practice, both with healthcare professionals and non-medical patients. Emphasis during medical training on acknowledging personal illness may help to challenge the ethos of invulnerability in the profession. illness'. This phrase was chosen to encompass psychiatric, physical, acute, and chronic, but not minor, illnesses. Encouraging the participation of individuals with a range of health concerns was a direct strategy to achieve maximum variation. The lead investigator ensured that all participants knew she was an experienced qualitative researcher from a non-medical background and that the local surgery supporting the research would not have access to any identifying information.

Seventeen GPs (10 male, 7 female) contacted the researchers in response to the e-mail invitation, and were then given information about the study either online or by post, according to their preference. All 17 GPs signed consent forms to demonstrate their willingness to participate in interviews. While interpretative phenomenological analysis advocates a maximum of 10 participants, the researchers felt that including all 17 responders would permit a more in-depth analysis and allow a novel and richly textured understanding of the diverse experiences of illness in GPs. ${ }^{18}$

\section{Collection and analysis of data}

The lead researcher carried out all the semistructured interviews at participants' own homes or in their surgeries, according to their preference. Interviews lasted 45-90 minutes and were guided by a schedule, designed with reference to the review of the literature..$^{3,5-13}$ The interview schedule was designed to elicit the experience of being a patient, whether being a patient has subsequently affected practice, and participants' reflections on giving care to and receiving care from other doctors. The interviews were audiotaped, transcribed verbatim, and anonymised through the removal of all identifying information. Participants were offered the opportunity to see the transcripts of their interviews prior to the analysis in order to verify their accounts.

The lead researcher engaged in an on-going process of reflexivity through making notes after each interview. This record of their own experience of the interview process was a useful point of reference during the analysis and enabled the researcher to engage in 'reflexive bracketing'. This is a process which involves the researcher developing a 'thoughtful, conscious self-awareness' 19 to bracket out, or at least identify, their personal suppositions. ${ }^{20,21}$ This process allows the researcher to reduce the influence of their own experience on the phenomenon under investigation. This process also ensured that interpretative accounts were elicited through a dynamic interaction between researcher and participant. ${ }^{17}$

The lead researcher was primarily responsible for the analysis and steps were taken at each phase to 
increase reliability. The research team agreed to exclude one researcher from the data analysis process to reduce possible bias arising from their own experience with training doctors in some of the areas relevant to this research. In line with the accepted analytical process for interpretative phenomenological analysis, ${ }^{22}$ each transcript was coded line by line into themes using NVivo 8 software. Similar themes from each transcript were then grouped and overarching categories were identified through an examination of the relationship between the themes.

The process of implementing interpretative phenomenological analysis is not simply concerned with the generation of themes. Meaning is central and the aim is to understand the complexity and content of those meanings, rather than to measure the frequency of themes..22 Thus, the researcher is required to engage in an interpretative relationship with the transcript: 'a sustained engagement with the text and a process of interpretation', whereby the researcher's role is to make sense of what the person is saying, while constantly checking their sensemaking is true to what the person actually said. ${ }^{22}$

Reliability was assessed in three ways. An exemplar transcript was read by every member of the research team and was then coded independently by both the lead researcher and an experienced qualitative researcher. Results were compared and there were no significant inconsistencies and so interpretative phenomenological analysis proceeded. The research team then reviewed the emerging findings individually before meeting as a group to discuss the structure of the categories. Participants were provided with a summary of findings to ensure that the interpretation reflected their own experiences.

\section{RESULTS}

\section{Participants}

Median age of participants was 46 years (interquartile range $36-58$ years). Participants were all qualified GPs, including locums, assistants, and partners. Some were retired, on maternity leave, or on sick leave. Significant illnesses reported by participants were: chronic medical conditions $(n=5)$; mental health conditions $(n=4)$; malignancies $(n=3)$; trauma $(n=3)$; surgical $(n=1)$; and undiagnosed condition $(n=1)$.

Data used to illustrate the findings are verbatim quotes which have been altered as little as possible to retain authenticity, while maintaining anonymity.

\section{General Medical Council recommendations}

Although a question asking whether or not participants had kept to the General Medical Council recommendation of being registered with a doctor from another practice was not included, this issue did come up naturally in most of the interviews. The majority of participants were following this recommendation, although two participants had consulted a doctor from their own practice (GP009 and GP0011).

\section{Who cares when doctors are ill? The 'caring profession' versus 'the system'}

The caring profession was described as 'a fold' (GP015) which should 'look after its own' (GP012) through a code of care based on understandings about professional courtesy. Some participants interpreted the latter as preferential treatment, although this was not felt to be elitist or inappropriate. When these expected codes of care were not met within secondary care some participants had to assert their status:

'I had to really pull rank in order to get my bed that day which I found a bit sad because nobody was saying "He's a doctor, you do something about it". Whenever I see patients who are related to the profession I always do my little bit extra.' (GP007)

Other GPs circumvented 'the system' to gain the most appropriate care for their condition. However, this was commonly a panic-based response, enacted only when they felt that the medical care they required was not provided. They acknowledged that referring themselves directly to secondary care or to a particular consultant contributed to a perception that doctors can be demanding or difficult patients. By contrast, others articulated strong views about this:

'I don't ever bypass the GP ... I would never go straight to a consultant for anything ... I think if you do that you get either under-treated or overtreated ... I think if you're going to get the best, most rational treatment you go through the system because the system is tried and tested.' (GP014)

The pressurised system that characterises primary care was a crucial factor predicting organisational problems of covering for sick colleagues. Not only did this pressure frequently lead to 'sympathy fatigue' (GP009) with sick colleagues but it was also deemed responsible for delays in seeking treatment:

'There's not much slack in the system so there's a great feeling that you've got to keep going and keep coping.' (GP012) 
The same participant advocated:

'Having a system where there's a bit of slack. But I realise that's never going to happen because (of) the pressures on the system.' (GP012)

Within 'the system' prevails a cultural code of invulnerability that relies on and encourages GPs to continue working through their own illness, or to return to work rapidly after sick leave. Tensions between acknowledging poor health and a prevailing sense of invincibility were evident in many accounts:

'I went back to work and struggled, really struggled, struggled with a full day, and [at] about 3 o'clock, because I [was] used to having siestas, I would be getting really, really tired and, but of course, I could do it, I can do illness.' (GP006)

Responders recognised the consequences and implications of returning to work too soon after illness. Many talked about their own struggles to cope when they had returned to work prematurely. They articulated the paradox of knowing what medical advice would advocate, yet by contrast acting on their own feelings that they should be able to cope with illness. This strong sense that they should be able to manage ('doing illness') led to them presenting a façade of coping, not just to their colleagues and patients but, more importantly, to themselves.

GPs who sought health care exemplified experiences of 'the system' ranging from flawed and inefficient to positive and trustworthy. Personal experiences of this system challenged the perspective of some participants:

'It just surprises me ... I'm seeing myself and the system as bad, but actually when I go as a patient it's good.' (GP004)

\section{Being a doctor-patient}

Among the participants a combination of personal and structural factors acts as a barrier to seeking health care, which resonates with the anxieties of non-doctors about the same issue:

'I found it difficult to recognise illness and to admit to illness and to seek medical advice: one, because it's difficult to get the time off; and, secondly, because of sort of ... the natural fear that you have "Oh God I don't want to find out something horrible".' (GP016)
Behaviours ranged across a continuum from reluctance to be a patient, through passivity, to total hand-over. Reluctant doctor-patients sometimes cited their personal disposition and the demands of working within primary care as barriers to acknowledging illness:

'I was busy, and I didn't want to be a patient and I didn't want to make a fuss.' (GP001)

The more passive doctor-patients were those who experienced illness that was either life-threatening or which physically prevented them from continuing to work. Some participants coped by disassociating themselves from their professional status:

'I just ... sort of wiped out the fact that I was a doctor during that time and just let other people look after me and that was my way of dealing with it.' (GP009)

Many of the participants identified tensions between being a 'good' patient and being demanding, and this related to the medical knowledge that doctor-patients have. A sense of being both 'us' (doctor) and 'them' (patient) was pervasive. Experiencing 'being a patient' and the illness process was referred to as being 'on the other side' (GP002) and this gave participants key insights into the experiences of their own patients, including fear, loss of control, uncertainty, hope, and despair. Experiencing disempowerment emerged as a particularly strong theme:

'You never really appreciate what it's like being a patient until you're a patient. And I think suddenly you realise that you feel very small and that you don't have much of a voice and you don't feel very powerful, and you feel very vulnerable and you can get very emotional, and you cannot be very rational about things.' (GP011)

Ironically, reflecting on their experiences of disempowerment actually appeared to give participants the skills to become stronger and more empowered. The reality of experiencing illness encouraged many GPs to recognise and become more accepting of their own limitations:

'I used to think sending people away and saying "Oh don't worry, it's nothing to worry about" was going to reassure them. But I know now that isn't the case and actually you still worry because you're not any better and nobody can tell you what it is, and you kind of think "Well how can 
you tell me it's not serious because you don't know what it is?". You know, so my doctor brain says "Well that's rational, that's fine, I know we don't know everything", but my person brain says "Oh no". You know if you're still feeling poorly you want someone to tell you what it is.' (GP0011)

Participants also reported that reflecting on their own feelings when in a vulnerable situation helped them to see how they could improve their consultation style. This appeared to be particularly so when doctor-patients experienced a 'bad' consultation:

'I think it's ... it's inevitable but you're much better at something when you've had experience of it. The other side. Um it's not that you should have to take every pill on the market and undergo every procedure ... but I think that actually being a patient and seeing how doctors are in the consultation in terms of how they share information, how they give information, how they appear in terms of the time that they might have, how in terms of listening ... it's all important. I think you learn ... you know once you've experienced a few bad examples of it ... it's much easier to see what you should be doing.' (GP003)

In the light of this, participants felt that it would benefit doctors if they were able to acknowledge their own vulnerability during training:

I think ... encouraging medical students to realise that they're only human, and doctors can get ill like anyone else and to ... seek help ... is a common-sense thing to do.' (GP012)

Participants' reports of their experiences of being a patient are in many respects similar to reports of non-doctor patients. What sets this group apart from non-doctor patients is the conflict of identity experienced by doctor-patients. These findings suggest that doctors who are unwell are likely to experience role ambiguity:

'I mean, if I knew what I thought was wrong with me l'd probably say "You know l'm a doctor and I think this is what I've got"... I want them to treat me just like any other patient and get that information ... So you want to be treated you know with a bit of professional respect 1 suppose, but you also want the same information that you would get from anyone else.' (GP011)

\section{Treating doctor-patients}

Of the 17 GPs in this study, 16 reported that they had experience of acting as physicians to other doctors. Participants were asked to reflect on these experiences and while perspectives were diverse, many participants articulated anxiety about treating other doctors:

'It's always really awkward ... particularly ... if that person is a friend or if it was somebody perhaps who was more senior to you and perhaps trained you, there's always other dynamics there, it's not just [a] patient-doctor relationship ... and it is more awkward; I don't think you consult in the same way, you're not as natural about it.' (GP011)

Doctor-patients were regarded by some participants as 'the same but different' (GP004) to non-medical patients and issues about shared language, trust, and safety were central to consultations. Negotiating shared care was difficult, and many participants illustrated how their experience of health care had helped them to recognise the importance of making no assumptions about the presenting patient:

'I think the more you look after doctors ... the more you realise that you should never make any assumptions, and sometimes it's helpful to have conversations with somebody at the outset about how they want to treat [doctor-patients].' (GP003)

'I think actually it's crucial they're treated as patients, they have the same fears, so in fact l'm quite scrupulous ... not just take for granted that they've considered things ... it's forging a middle way ... between taking things for granted and ... yet allowing them to be vulnerable.' (GP006)

In particular, the experiences described by the doctor-patients demonstrated that their feelings of vulnerability had not always been taken into account, thereby highlighting the fragility of balancing shared care:

'Some of them will pitch it at you as a fellow doctor, but not as a patient, often when you're actually needing it as a patient ... and that can be quite brutal in a way.' (GP008)

The experience of role ambiguity was therefore recognised as being challenging for both the doctors who were treating fellow doctor-patients and those doctor-patients who had received treatment. The experience of serious illness meant that these 
participants felt able to draw from their own experiences and, therefore, reduce the potential for role ambiguity in future interactions with other GPs. However, for some, making the transition from role to role was challenging. There was also a general recognition of the need to 'go through the system'. In particular, the role of informal consultations was considered by some to be an inevitable occurrence within this professional group, but the dangers of relying on informal health care were highlighted. One participant reflected that since their illness, they had registered with a GP outside their own area:

'I feel sort of better looked after because ... before it was much more a kind of shared responsibility ... if you discuss something in the corridor or after surgery or whatever it's not the same as actually going along to somebody specifically and saying "Look what do you think l ought to do about this?" ... I think I just feel more comfortable ... it's the decision making ... and the weighing up things and doing that objectively.' (GP009)

\section{Impact on practice}

Through their illnesses, the GPs had become aware that they had experienced many similar emotions to their patients and this often led to sympathy with the predicament of patients and to an increased sense of empathy:

'I was ill and I was having to fight my case and if I had been a patient without any status, I'd have had to have waited and I felt very sorry for my patients, that's what I kept thinking, how impotent they feel.' (GP006)

Recognising the sense of disempowerment experienced by many patients prompted some GPs to adopt strategies to address this; for example, by making facilitating statements to encourage patients to ask questions or to express their concerns. Other GPs modelled strategies used by the doctors that they had consulted when they were ill, to empower their own patients:

'I use a lot of techniques that my therapist uses when I see patients... just, you know, stay in silence for a second ... just a few seconds longer makes all the difference otherwise you're jumping in with solutions and things like that ... you know it's all very powerful sort of techniques.' (GP004)

The role of empathy was important in predicting alterations to both the GPs' attitudes and their practice. Participants suggested that while empathy is difficult to teach in a training environment, there are strategies to facilitate understanding of disempowerment and vulnerability. One GP said:

'Sitting in my [own GP's] surgery, I always feel like I'm waiting outside the headmaster's office ... [trainees could reflect on times] when you had to see someone you felt had authority, how did that make you feel? ... most of us have got some kind of thing we can draw on like that even if it's not medically related.' (GP011)

Participants reflected on the costs and benefits of using self-disclosure in consultations. Some articulated that sharing information about their own experiences can, in certain situations, be a helpful consultation tool. By contrast, others felt that this would 'alter the rules of engagement' (GP004), and might constitute an abuse of trust within the doctor-patient relationship. In both cases, selfdisclosure could be seen to change the personal dynamics of the consultation:

'That's about giving them a bit of yourself which you do as a doctor in all sorts of ways; that's a very direct way of doing it ... so I think, yeah, sharing it with patients ... in the right time and the right place can be a helpful thing.' (GP003)

Therefore, assessing the costs and benefits of selfdisclosure was an issue which required careful consideration of both the needs and potential reaction of the individual patient:

'I think you have to be wise about who you share things like that with, um, and there are some patients who find it really helpful, it's really beneficial but there are some people who I know it wouldn't be terribly wise to open oneself up in that way because they might actually ... not abuse it but just take advantage of it inappropriately. So I think you must be a bit careful about sharing that sort of information, but for some patients I think it ... it is really quite helpful, quite useful.' (GP012)

Practical changes to consultation style were made on the basis of the participants' own experiences of illness and treatment. Attitudes to and rates of referral often changed according to personal experience:

I think I'm much more inclined ... to hassle maybe on a patient's behalf ... to get things done.' (GP017)

'I think I'm slightly more appreciative of ... how reassuring it can be to have ... the fears that 
aren't particularly sort of likely put at rest. So I don't know, maybe l'll end up sort of consuming more NHS resources.' (GP013)

Some participants noticed a qualitative change in their communication style while others reflected on their greater connection with patients:

'I actually felt I was more involved as in I ... cared more ... that sounds a bit ... nebulous, but actually it did ... it made more sense what the people were coming in with ... I could see why they were as distressed as they were ... and I could relate to it more.' (GP015)

Thus a key change in the practice of this study's participants was a more active involvement with their patients. This involvement was both on a practical and emotional level. Participants reported chasing up appointments on their patients' behalves, and of offering more tests to patients. Participants also spoke of a more active emotional involvement because they felt better able to connect with their patients' experiences. This arose through the identification of an area of communality, which removed a barrier of distance between doctor and patient and thereby increased understanding.

\section{DISCUSSION}

\section{Summary of main findings}

This study's findings confirm that the ethos of invulnerability to illness among doctors ${ }^{13}$ is established through factors including personal disposition and medical knowledge. In addition, the study has shown that this culture is maintained by the pressurised environment that characterises general practice. ${ }^{23}$ This powerful combination prevents many GPs from seeking medical attention or from taking time off work. This research responds to the recent call for evidence to support the volume of anecdote and opinion about the barriers to health care for doctors $^{4}$ and has implications for both the care of doctors and for further areas of study. These findings show that greater consideration needs to be given to address the barriers to health care for doctors who become patients, such as lack of time, feelings of disempowerment, and role ambiguity.

\section{Strengths and limitations of the study}

While researchers who employ interpretative phenomenological analysis methodology often advocate including up to 10 participants, Smith and Osborn $^{22}$ acknowledge that the question of sample size depends on several factors including 'the richness of the individual cases', and they highlight that studies have been published with samples of 15 participants. In this study, 17 GPs offered to participate and the researchers felt that this would give an insightful and diverse range of experiences about the phenomena of illness in GPs without compromising the integrity of the analytic process.

The term 'significant illness' may appear vague but was intentionally used in recruitment material to promote the participation of any GPs who considered their illness to be important in their own lives. It was acknowledged that on this basis participants were self-selecting and that some may have been motivated by either a very good or very poor experience of health care.

The seven participants who requested to see their interview transcript prior to analysis made grammatical amendments and a few asked for small sections to be excluded to reduce the possibility of identification. While all the participants were provided with a summary of the findings for comment, only one did so, and agreed with the authors' interpretation.

This study reports the participants' own perceptions of how personal illness affected their practice, although future research might take a more objective approach to measure actual changes.

\section{Comparison with existing literature}

These findings offer evidence of the complex and uncomfortable phenomenon both of treating and being 'doctor-patients'. The British Medical Association recommends that to foster a productive doctor-patient relationship, doctors who are receiving medical care should acknowledge that they have become the patient in the partnership. ${ }^{24}$ However, this study's findings demonstrate that while some participants willingly handed over their care, others were reluctant to accept the patient role. The fragility of these consultations and the inherent sense of role confusion indicate that GPs could benefit from components within medical training on how to 'be a doctor to a doctor', ${ }^{15}$ as well as 'how to be a patient'. Further research is needed to show the best way to integrate these issues into medical training and continuing professional development. This should help to encourage GPs to acknowledge their own health needs, as well as those of their colleagues. This in turn may help to reduce levels of anxiety when treating colleagues.

\section{Implications for future research and clinical practice}

Strategies to address the cultural code of immunity to illness need to be delivered at both an individual and organisational level. First, during training medical professionals should be encouraged to explore and acknowledge the potential failings of their own health. Second, primary care organisations should be 
encouraged to help GPs offset the organisational difficulties of sick-leave, thereby altering the organisational structures that perpetuate the pressure for doctors to deny ill health. ${ }^{4}$ Greater flexibility in general practice culture is needed to accommodate doctors who cope with chronic physical and mental illnesses, including flexible career and training options. ${ }^{25}$

It is recommended that strategic guidelines be developed for practices and primary care organisations, based on a model of occupational health for primary care. ${ }^{26}$ Such guidelines need to provide validation that illness does not belong only to patients, ${ }^{5}$ and should also offer procedural advice to ensure that GPs who are sick are looked after within an efficient and caring system. Recognising and coping with illness in GPs might also address factors contributing to poor performance in doctors. ${ }^{27}$

Following a period of significant illness, GPs in the current study frequently experienced increased empathy with their patients. However, it was found that the experience of being a doctor-patient had little other effect on subsequent care for participants' own patients. Previous research identifies that doctors who have been unwell report improved communication with their patients,,$^{16}$ and the current research highlights specific strategies to empower patients within consultations.

The findings of this study suggest that the process of being a doctor-patient is complex and that experiences of the current healthcare system do not always match expectations of receiving treatment from a 'caring profession'. Following a period of illness, GPs may alter strategies used in consultations with both non-doctor patients and doctor-patients. The issues of empathy and empowerment have implications for the training and development of doctors. The prevailing ethos of immunity to illness should be challenged, both at the individual and organisational level. These findings consolidate existing anecdotal evidence about illness among the profession and should stimulate future research and debate about occupational health issues within primary care.

\section{Funding}

This study was funded by the Pan Bath \& Swindon Primary Care Research and Development Consortium (2007/092). The authors' work was independent of the funders who had no involvement in the research process

\section{Ethical approval}

Ethical approval for this study was granted by Gloucester NHS Research Ethics Committee (08/H0105/4)

\section{Competing interests}

The authors have stated that there are none

\section{Acknowledgements}

We thank the GPs who took part in this study, Christina Maslen for help with the literature review, and Emma Clarke for her support at Hope House Surgery.

\section{Discuss this article}

Contribute and read comments about this article on the Discussion Forum: http://www.rcgp.org.uk/bjgp-discuss

\section{REFERENCES}

1. Parsons J. Are doctors immune to depression? Aust Fam Physician 2001; 30(3): 225-231.

2. British Medical Association. Work-related stress among senior doctors London: BMA, 2000.

3. Allibone A. Who treats the doctors? Practitioner 1990; 234(1496): 984-987.

4. Kay M, Mitchell G, Clavarino A, Doust J. Doctors as patients: a systematic review of doctors' health access and the barriers they experience. Br J Gen Pract 2008; 58(552): 501-508.

5. McKevitt C, Morgan M. Illness doesn't belong to us. J R Soc Med 1997; 90(9): 491-495.

6. Thompson WT, Cupples ME, Sibbett $\mathrm{CH}$, et al. Challenge of culture, conscience and contract to general practitioners' care of their own health: qualitative study. BMJ 2001; 323(7315): 728-731.

7. Richards C. The health of doctors. London: King Edwards Hospital Fund, 1989.

8. Richards JG. The health and health practices of doctors and their families. NZ Med J 1999; 112(1084): 96-99.

9. Rosen IM, Christie JD, Bellini LM, Asch DA. Health and health care among housestaff in four US internal medicine residency programs. $J$ Gen Intern Med 2000; 15(2): 116-121.

10. Shadbolt NE. Attitudes to healthcare and self-care among junior medical officers: a preliminary report. Med J Aust 2002; 177(Suppl): S19-20.

11. Fromme E, Hebert R, Carrese J. Self-doctoring: a qualitative study of physicians with cancer. J Fam Pract 2004; 53(4): 299-306.

12. Chambers R, Belcher J. Self-reported health care over the past 10 years: a survey of general practitioners. Br J Gen Pract 1992; 42(357): 153-156.

13. Stein H. American medicine as culture. Boulder, CO: Westview Press, 1990.

14. Ingstad B, Christe V. Encounters with illness. The perspective of the sick doctor. Anthropol Med 2001; 8(2/3): 201-210.

15. Jaye $\mathrm{C}$, Wilson $\mathrm{H}$. When general practitioners become patients. Health 2000; 7(2): 201-225.

16. Klitzman R. Improving education on doctor-patient relationships and communication: lessons from doctors who become patients. Acad Med 2006; 81(5): 447-453.

17. Smith JA, Jarman M, Osborn M. Doing Interpretative phenomenological analysis. In: Murray M, Chamberlain K (eds). Qualitative health psychology: theories and methods. London: Sage Publications, 1999: 218-240.

18. Sandelowski M. Focus on qualitative methods. Sample size in qualitative research. Res Nurs Health 1995; 18(2): 179-183.

19. Finlay L. 'Outing' the researcher: the provenance, process, and practice of reflexivity. Qual Health Res 2002; 12(4): 531-545.

20. Hutchinson SA. Grounded theory: The method. In: Munhall PL, Boyd $\mathrm{CO}$ (eds). Nursing research: a qualitative perspective. 2 nd edn. New York: National League of Nursing, 1993: 180-212.

21. Kvale $S$. The qualitative research interview: A phenomenological and hermeneutical mode of understanding. J Phenomenol Psychol 1984; 14(2): 171-198.

22. Smith J, Osborn M. Interpretative phenomenological analysis. In: Smith J (ed.). Qualitative psychology: a practical guide to research methods. 2nd edn. London: Sage Publications, 2008: 53-80.

23. Smith R. All doctors are problem doctors. [Editorial] BMJ 1997; 314(7084): 841-842.

24. British Medical Association. Ethical responsibilities in treating doctors who are patients. Guidance from the Ethics Department. March 1995. Revised March 2004

http://www.bma.org.uk/images/Doctor\%20patients\%20new\%20layout \%202_tcm41-147309.pdf (accessed 9 Oct 2009).

25. Jones P. Doctors as patients. Oxford: Radcliffe Publishing, 2005.

26. Forsythe M, Calnan M, Wall B. Doctors as patients: postal survey examining consultants and general practitioners adherence to guidelines. BMJ 1999; 319(7210): 605-608.

27. BBC Radio 4. Doctors in difficulty. 2008 July. http://www.bbc.co.uk/radio4/science/allinthemind 20080708.shtml (accessed 21 Sep 2009). 